\title{
MEMÓRIA E MORTE: UMA ABORDAGEM DA FAMÍLIA ROMANA POR MEIO DA CULTURA MATERIAL
}

\author{
Luciane Munhoz de Omena ${ }^{1}$
}

\section{RESUMO}

Esse artigo tem como objetivo compreender as representações da morte e suas relações com a memória a partir da estela funerária de Ursilialngenua. Para tanto, traçaremos algumas reflexões críticas acerca das dimensões mais particulares e emocionais da lembrança dos mortos, à medida que o túmulo poderia se tornar um espaço de peregrinação e reverência, uma casa ou santuário para os mortos e modo de associação dos mortos com as divindades e seus atributos. Conforme nossa análise, tais reminiscências mantiveram não apenas o nome de Trebio vivo, mas cumpriram as necessidades emocionais dos sobreviventes.

Palavras-chave: Morte, Memória, Família e Dor

\section{ABSTRACT}

This article aims to understand the representations of the death and its relations with the memory from the funerary stele of Ursilialngenua. To do this, we will make a few critical reflections about the most private and emotional dimensions of remembrance of the dead, as the grave could become a place of pilgrimage and reverence, a house or shrine for the dead people and connection of the dead people with gods and their attributes. According to our analysis, such reminiscences maintained, not only the name of Trebius alive, but it satisfied the emotional needs of survivors.

Keywords: Dead, Memory, Family and Sorrow

\section{RESUMEN}

Este artículo tiene como objetivo comprender las representaciones de la muerte y su relación con la memoria de la estela funeraria de Ursilialngenua. Nos basamos a cabo algunas reflexiones críticas sobre las dimensiones más privadas y emocionales de la memoria de los muertos, como la tumba podría convertirse en un lugar de peregrinación y de reverencia, una casa o un santuario para el modo de muertos y de asociación de los muertos con las deidades y sus atributos. Como nuestro análisis, tales recuerdos no sólo mantuvieron el nombre vivo Trebio, pero cumplen las necesidades emocionales de los sobrevivientes.

Palabras clave: Muerte, la memoria, la familia y el dolor

\footnotetext{
${ }^{1}$ Professora Adjunta de História Antiga da Universidade Federal de Goiás\Brasil. Pós-doutoranda no Instituto de Filosofia e Ciências Sociais UUNICAMP sob a supervisão do Prof. Dr. Pedro Paulo A. Funari. Bolsista CAPES. E-mail: lucianemunhoz34@gmail.com

\begin{tabular}{|l|l|l|l|l|l|l|}
\hline (c) Rev. Arqueologia Pública & Campinas, SP & v. 9 & n. 2 & p. 19-29 & DEZ. 2015 & ISSN 2237-8294
\end{tabular}
}




\section{Revista de Arqueologia Pública}

\section{Morte e materialidade no mundo romano}

Ao levarmos em consideração a relevância das representações mortuárias no Mediterrâneo Ocidental, investigaremos, neste artigo, a partir de uma estela funerária, localizada na Via ManzoniGiardino, em Milão (Museo Archeologico di Milano, N. Inv. A 0.917767), o papel da produção de uma memória pública que colocava em evidência não somente os símbolos e as insígnias que projetavam o morto na estrutura de poder e em sua posição nas gerações da família, mas também nos concentraremos nas impressões mais particulares e emocionais da lembrança dos mortos. Sabemos, pois, que a morte representava uma experiência social, à medida que englobava a forma como os mortos na sociedade romana passavam a ser lembrados ou, dependendo das circunstâncias, condenados ao esquecimento. Partimos do pressuposto de que a memória vinculava-se à produção social, pois, parafraseando Hope (2005: 115), a pena capital, por exemplo, envolvia não somente a execução do indivíduo, mas também a decapitação, a exposição do corpo, o abuso do cadáver e a negação de sepultamento. A partir disso, podemos afirmar que o corpo inanimado tornou-se símbolo de poder, não pela sua condição de passividade, tal como explora Hope (2000: 104-127), mas por ser um objeto poderoso e simbólico, à medida que poderia ser honrado, valorizado ou desprezado. A materialidade do corpo criava significados individuais e sociais, pois, assim como argumentamos, a morte e, por consequência, o morto tornaram-se parte de um espetáculo público. Podemos perceber que, em função das punições públicas, tal como a crucificação em corpos vivos, os cadáveres normalmente eram marcados, mutilados, espancados e, em muitas circunstâncias, apodreciam na cruz. Em Petrônio, os corpos crucificados dos ladrões de Éfeso eram guardados por soldados para impedir a sua remoção. Neste sentido, as execuções púbicas ocorriam em assentamentos próximos às necrópoles e, conforme a compreensão de Hope (2000: 112), o contraste entre corpos putrefatos e sepulturas deveria impressionar os viandantes e, em nossa percepção, este fato produzia um cenário de desonra, um exemplum de punição institucional.

Criam-se, então, estratégias para a promoção de indivíduos e de suas póstumas memórias que, vinculadas à reputação, projetavam suas imagens em vestígios materiais e narrativas textuais (e.g. moedas, epitáfios e monumentos funerários, discursos históricos e filosóficos entre outros mais). A promoção individual na sociedade romana alicerçava-se, em termos explícitos, em uma publica memoria ou, dito de outro modo, em uma memória compartilhada (GOWING, 2005: 15). Sem sombra de dúvida, a paisagem funerária exercia um papel público na promoção de memória, incorporando conflitos e competições presentes na sociedade. O túmulo piramidal de Caius Cestius Epulo, membro do conselho sacerdotal, construído durante o Principado de Augusto, entre 18 ou 12 a.C., apresenta uma base de $29,5 \mathrm{~m}$ e mais de $36 \mathrm{~m}$ de altura, sendo o concreto revestido com blocos de mármore branco. A própria localização do túmulo tornava-se privilegiada, à medida que se encontrava na Via Ostiense, uma estrada movimentada, com acesso ao porto de Roma. Nesse sentido, em função do grande contingente de pessoas que, provavelmente, transitavam nessas estradas, encontramos na base superior da pirâmide - a leste e a oeste - a inscrição com o nome de 


\section{Revista de Arqueologia Pública}

Caius, e no lado leste, a inscrição informa aos transeuntes que, sob a vontade do falecido, a família ergueu o túmulo piramidal em 330 dias, a encargo de seu herdeiro Lúcio Ponto Mela, filho de Públio da família Cláudia e seu liberto Poto (OPVS APSOLVTVM EX TESTAMENTO DIEBVS CCCXXX ARBITRATV L. PONTI PF CLA. MELAE HEREDIS ET POTHI L.). Ademais, temos ainda informações sobre o exercício de seus cargos políticos (C C CESTIVS - L F F POB - EPVLO - PR - TR - PLVII . $V I R \cdot E P O L O N V M)$ e, respectivamente, na câmara mortuária, encontramos representações da deusa Vitória, incluindo vasos cerimoniais, que continham em sua estrutura interna painéis brancos com as representações da divindade alada, seguindo os parâmetros romanos.

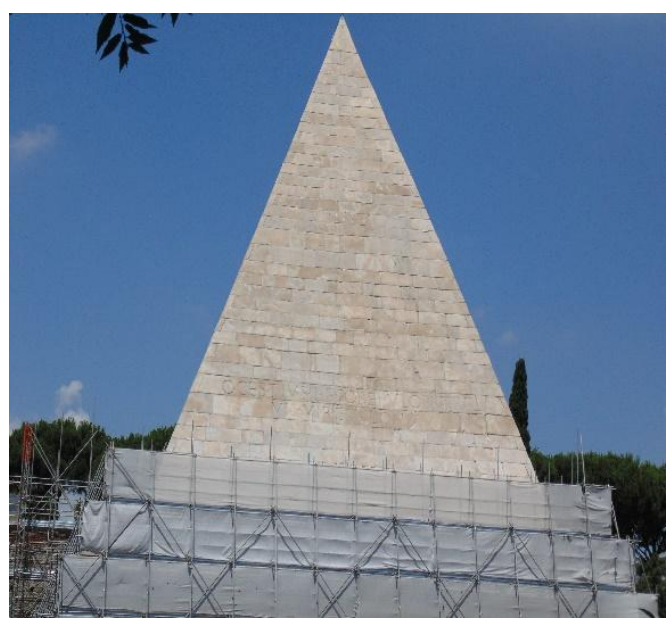

Fig. I Pirâmide de Cestius. Créd. Thiago E. A. Mota, 2014.

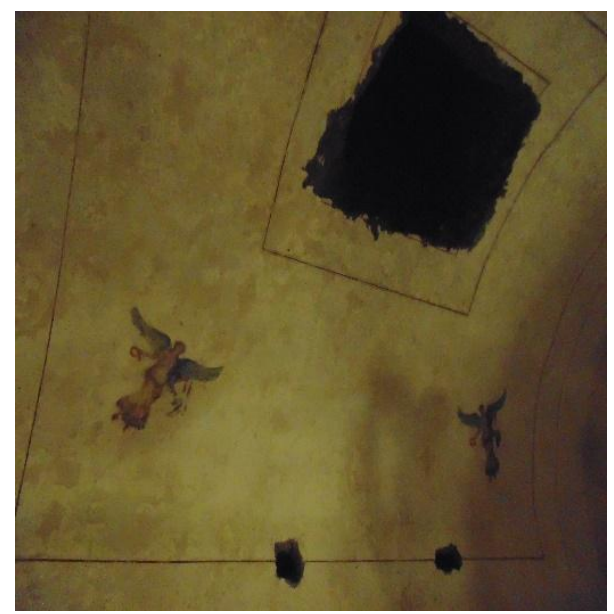

Fig. Il Parte Interna da Pirâmide de Cestius. Créd. Thiago E. A. Mota. 2014.

Queremos, com isso, enfatizar não somente a imponência da pirâmide, mas supor, sobretudo, o possível envolvimento de Cestius ou seus familiares nos conflitos políticos entre a domus de Augusto e Marco Antônio. Augusto e seus partidários (e.g. Tito Lívio, Horácio, Virgílio entre outros) (Cf. WOOLF (a), 2009: 315) difundiram imagens de protetores do costume dos ancestrais 


\section{Revista de Arqueologia Pública}

(mosmaiorum) e, em função disto, transformaram os egípcios em inimigos públicos de Roma e, de maneira indireta, a proximidade entre Marco Antônio e Cleópatra (Cf. EDER, 2005; GALINSKY, 2005; ROWE, 2013). O interessante desta oposição são os testemunhos que, em sua totalidade, se perderam ou foram silenciados, tornando, dessa forma, a pirâmide de Caius Cestius Epulo um relevante vestígio para se compreender a presença de conflitos. Por exemplo, em relação aos grupos de oposição à domus de Augusto, aparecem, de modo geral, somente em vestígios posteriores, tais como Veleio Paterculo, que menciona, de modo geral, aristocratas como Crispino, A. Cláudio e Demóstenes, os quais, aliados a Júlia e Julho Antônio, filho de Marco Antônio, opuseram-se aos direitos sucessórios dos filhos de Lívia, em especial, Tibério; assim como também hostilizaram-se à política senatorial para estabelecer a monarquia (Cf. HIDALDO DE LAVEGA, 2012: 27; CENERINI, 2009). Ao ser descoberta a oposição, em um processo judicial no Senado, Júlia, em 2 a.C., recebe a punição do exílio, a partir da Lex lulia de Adulteriis (Suetônio. Augustus 64, 4), promulgada, provavelmente, em 18 a.C., enquanto, Julho Antônio foi condenado à morte.

Ora, a construção do túmulo piramidal de Caius Cestius coloca em evidência, de fato, a referência à cultura egípcia e sua proximidade à domus de Marco Antônio, afastando-se, por consequência, das construções circulares, tal como no Mausoléu de Augusto e Cecilia Metella, ornamentados com guirlandas e cabeça de touro. É relevante mencionar que os vestígios com representações da cultura egípcia estavam presentes em todo o imperium como o obelisco ou mesmo a domus tardo republicana, localizada no Palatino, com uma decoração pictórica com motivos isíacos - flores de loto, pétalas de rosas, disco solar e a serpente - aparece também, em uma vertente clássica, painéis com fundo branco (Cf. CONSTANTINO, 2014). Sabemos, pois, que no espaço doméstico as escolhas e os usos ornamentais estavam mais livres e não se encontravam atrelados ao monitoramento pontificial (e.g. a exigência res religiosa da sepultura) (GENZOR, 2007: 261).

Todavia, se levarmos em consideração o discurso de divulgação de Augusto, a partir de autores como Tito Lívio (AbVrbe Condita I, XLIX) e Horácio (Carmina II, 2; 15; 16; III, 1), acerca da ancestralidade que se confrontava à ambitio e a superbia dos comportamentos, entendemos que 0 uso indiscriminado de adornos orientalizantes, poderia afastá-lo publicamente do mosmaiorum, pois, em nossa percepção, a domus imperial não representava somente um espaço físico, mas também, em termos simbólicos, promovia à Res Publica. Assim, a construção do Mausoléu de Augusto representava, em nossa percepção, uma criação identitária (Cf. HALES, 2009) que, em sentido explícito, o associava ao mores (legitimação da tradição) no espaço sagrado da urbs e indicava, ao mesmo tempo, a partir do discurso de ancestralidade, um meio de persuasão que se inseria em situações de conflitos (WALLACE-HADRILL, 2008: 225).

Em razão disso, os monumentos mortuários e a simbologia da morte, como produção de memória social, celebravam os sucessos familiares, a própria aristocracia e os sistemas de governo, pois, como argumenta Polo (2007: 94), "a celebração da morte envolveu um espírito cooperativo na aristocracia romana, uma vez que os falecidos da sua família e da comunidade continuariam 


\section{Revista de Arqueologia Pública}

eternamente na memória coletiva". Ao expressar reputação e glória, o monumento piramidal de Cestius, além de incorporar à imortalidade, marca a sua posição política e identitária, à medida que o modelo tumular associava-se às práticas mortuárias de enterramento egípcio. Entendemos que as construções identitárias são, sobretudo, relacionais e marcadas por suas diferenças e símbolos. Seguindo esta linha de raciocínio, os vestígios mortuários, a partir de narrativas textuais e materiais, posicionavam, em vias de fato, a aula romana em uma arena política representando hierarquias e conflitos sociais que, em termos efetivos, incidiam no tratamento dado ao corpo, no sexo em associação com o status social, a idade do falecido, sendo, tais elementos, incorporados à procissão funerária e aos tipos de construções, pois a necrópole representava um espaço de memória e, portanto, um espaço de transmissão.

A transmissão de uma memória subsiste em situações pontuais, em efetivo, em grupos sociais, pois, citando Candau (2011: 49), "toda memória é social, mas não necessariamente coletiva". Tem-se, então, duas festividades dedicadas aos mortos: a lemuria e a parentalia. Na primeira, a comemoração abarcava os mortos que, em função da ausência de sepultamentos, tornavam-se errantes, enquanto a parentalia destinava-se aos mortos que viviam na aeternadomus (Cf. WALLACE-HADRILL, 2008: 42). Realizada em fevereiro, com nove dias de comemoração, a parentalia criava a oportunidade para os indivíduos lembrarem de suas respectivas famílias, com a oblação de oferendas em túmulos, a rememoração do nascimento e morte do falecido, bem como a manutenção da tumba e, em especial, a continuidade do nome do morto e de sua família.

Como podemos notar, a festa, como produto da realidade social, exprimia conflitos, tensões, censuras e estruturas de poder que, vinculadas ao poder imperial, promoviam ações que idealizavam os mortos, celebravam a memória dos familiares e, em função disto, promoviam distribuições de dinheiro, alimentos e bebidas, além de espetáculos gladiatoriais; com isso, a produção da parentalia gerava imagens de generosidade da aristocracia romana (HOPE, 2003: 117).

\section{EPIGRAFIA E OS LAÇOS FAMILIARES EM COROGUENATE}

Sabemos, à vista disso, que as representações de afetividade foram igualmente mediadas pela interposição de documentação textual e material. Vale ressaltar que entendemos cultura material como sendo um conjunto de objetos, estruturas arquitetônicas, paisagens cultural e alimentar, assim como os próprios textos em sua condição física, à medida que consideramos a materialidade essencial para a compreensão das relações sociais. Partindo dessas premissas, a epigrafia aparece, segundo Woolf (2009: 57), "em formatos padronizados reduzindo, de fato, o número de palavras necessárias que seriam apresentadas aos espectadores, semelhantes aos ideogramas. Muitas lápides, por exemplo, proclamavam o assunto, mesmo antes da palavra lida". 0 que nos leva a concordar com Bersani (2014: 13), ao afirmar que a epigrafia transformava-se em veículo de comunicação, direcionando, em alguns casos, questões da vida pública em discursos 
celebrativos. Assim, as inscrições enalteciam o imperium, famílias aristocráticas, ou mesmo expressões de sofrimento decorrentes das mortes de familiares. Como acentua Hope (2003: 119),

todos os monumentos foram projetados para resistir e para perpetuar a memória, mas vale lembrar que eram construções seletivas, as quais representavam pessoas e informações de uma determinada maneira. Da mesma forma, podem ter sido enraizados em uma memória individual, mas, em última análise, os monumentos poderiam contribuir mais para a memória social. Memórias podem ser gravadas, literalmente inscritas, para os monumentos, mas a sua presença física e o fato de que se tornaram focos de rituais, amarra-os à memória social e o processo contínuo de como o passado é reformulado para atender as necessidades da vida.

As imagens da morte representavam um mecanismo de reprodução social, um veículo que se traduziria em uma produção discursiva controlada, selecionada, organizada e distribuída à sociedade (Cf. FOUCAULT, 1998). O repertório iconográfico e textual em estelas funerárias, ou mesmo em sarcófagos, tendiam a ser repetitivos e convencionais, indicando, dessa forma, um compartilhamento de ideais e valores comuns (BIRK, 2011: 29), que sinalizavam, sobretudo, representações de homens, mulheres e crianças, as quais incorporavam a cidadania romana (HUSKINSON, 2007: 325). Nesse percurso seletivo, destacamos que, em algumas circunstâncias, a escolha do repertório visual e textual não representava o indivíduo, pois, como acentua Birk (2011: 230), "a família poderia, através da compra de um sarcófago de um parente falecido, construir uma identidade que, ao final, também refletia a própria família".

Conforme temos visto, os túmulos encontrados na Via Manzoni localizam-se, de modo geral, entre os séculos I a.C. a IV d. C. Segundo Coparusso (2012: 130), as sepulturas continham ossos queimados dentro de urnas de cerâmica, ventro ou ânforas soterradas ou, em algumas situações, colocadas junto com os objetos funerários em caixas e, mais raramente, em lajes de pedra. Além disso, as sepulturas comportavam objetos simbólicos tais como "moeda, lâmpada, pote para unguento perfumado e, em sua grande maioria, incluíam vários recipientes de vidro, inclusive, alguns deles coloridos, serviço de mesa de cerâmica e também objetos preciosos em bronze" (COPARUSSO et al, 2012: 130). Podemos identificar uma riqueza de vestígios mortuários que se concentram quase, em sua totalidade, no Museo Archeologico di Milano, com uma quantidade expressiva de altares funerários com narrativas escrita e imagética. 


\section{Revista de Arqueologia Pública}

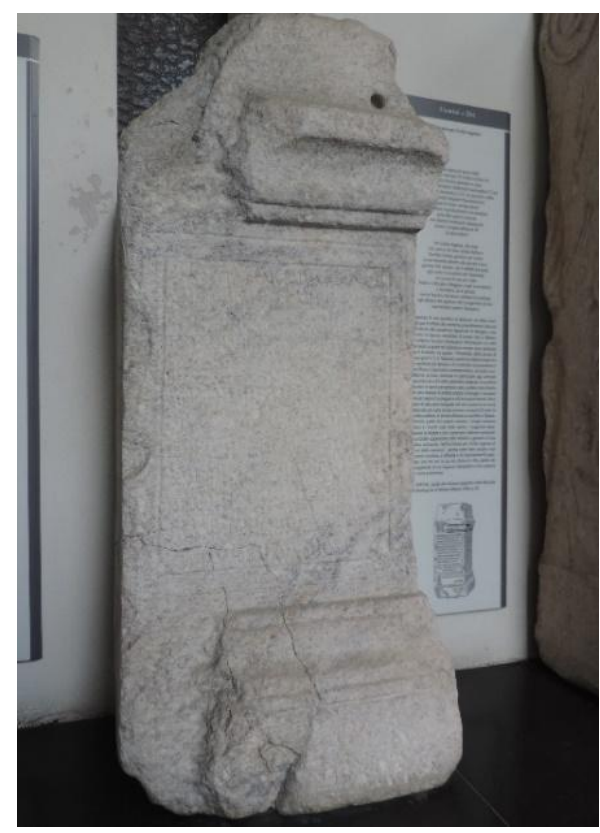

Fig. III Estela Funerária de Ursilialngenua

Museo Archeologico di Milano. Crédito: Luciane Munhoz de Omena, 2014.

Devemos enfatizar de tal modo que, os rituais mortuários e suas celebrações fundamentavam-se na imortalização do falecido, levando-se em consideração a construção de ideais que, em nossa opinião, estimulavam o papel público de seus familiares. Por exemplo, temos uma estela funerária, localizada na Via Manzoni-Giardino, em Milão (Fig. III Museo Archeologico di Milano, N. Inv. A 0.917767 apud SARTORI, 1994), dedicada a Ursilialngenua. Seus pais Ursilius Rufinus e Domitia Severa dedicaram a estela à memória de sua filha, em um altar funerário de mármore, medindo $86 \mathrm{~cm}$ de comprimento, $40 \mathrm{~cm}$ de largura e $27 \mathrm{~cm}$ de espessura, localizado na necrópole de Milão e anexado, segundo Sartori (1994, p. 82), provavelmente na parte interna do complexo sepulcral da família, entre os séculos II - III d.C. Ao morrer com oito anos e seis meses (Museo Archeologico di Milano, N. Inv. A. 0.9.11034. Linhas 1 e 2 apud SARTORI, 1994)-, não somente garantiu a perpetuação de sua memória na estela funerária, como também fez com que seus pais disponibilizassem 400 sestércios aos jovens, com os quais deveriam colocar três coroas de rosas no túmulo de Ursilia à época da Parentalia; assim como em seu aniversário, estariam incumbidos das libações (Museo Archeologico di Milano, N. Inv. A. 0.9.11034. Linhas 4, 5, 6, 7, 8 e 9 apud SARTORI, 1994).

Diante destas considerações, a inscrição epigráfica de Ursilia apresenta o túmulo como sendo um espaço de peregrinação e reverência, uma casa ou santuário para os mortos e as associações dos mortos com as divindades e seus atributos mantiveram não apenas o seu nome vivo, mas também a presença de seus familiares no monumento mortuário. Ao que nos parece, o ambiente festivo, incorporado às doações de alimentos, banquetes, ou reparações nos túmulos, demonstrava não apenas a produção de memória do morto e de seus familiares; porém, em função 


\section{Revista de Arqueologia Pública}

das obrigações religiosas dos homens para com os deuses, temos igualmente à associação da comunidade com os espíritos dos mortos, que, aparecem nas estelas funerárias seguindo a fórmula DM, Dis Manibus.

A partir da observância dos rituais - relegere - se não fosse devidamente cumprida pelos jovens, a comunidade de Coroguenate deveria depositar as coras de rosas no túmulo de Ursilia. Ainda que não tenhamos a idealização da morte infantil no espaço doméstico com familiares, ou mesmo cenas convencionais de heróis e divindades, que apareciam, geralmente, em sarcófagos e em relevos (Cf. HUSKINSON, 2007), o epitáfio de Ursilia acentua os laços da família nuclear, com o conjunto entre a criança e os pais, expressando, em nossa percepção, a produção de uma memória que, associada à preocupação monetária dos pais, exaltava, em termos simbólicos, as relações de afetividade e de pietas familiar.

\section{CONSIDERAÇÕES FINAIS}

As representações mortuárias na sociedade romana, mesmo que não correspondam à totalidade da população, nos estimula, como temos percebido, às reflexões sobre as relações estabelecidas com os elementos políticos, sociais, econômicos, culturais e simbólicos. Em nossa leitura, a produção de memória social vinculava-se à promoção de imagens, ou mesmo à destruição da reputação de um indivíduo a partir da remoção de estátuas e imagens; além disso, tal produção associava-se igualmente à exclusão do nome nas inscrições públicas, a proibição da recordação da família, à medida que, em alguns conflitos na aula romana, em especial o crime de lesa majestade, proibia-se o luto e a exposição da imagem do condenado em futuros funerais da família. Todos estes aspectos, aliados à extensão pública da dor, poderiam levar homens e mulheres não apenas à morte física, mas, em especial, a morte social. Com este viés interpretativo, as cerimônias públicas como parentalia e lemuria relacionavam-se às formas de comunicação social e política. As celebrações convertiam-se em mecanismos de manipulação da opinião pública, por meio de imagens a legitimar o mando ou, assim como entendemos, a expressar conflitos e hierarquias sociais. Em razão disto, entendemos que, mesmo em nome dos mortos, o epitáfio de Ursilia Ingenua convertia-se em práticas discursivas. Com isso, os monumentos mortuários dedicados aos familiares e indivíduos expressavam, com muita nitidez, construções identitárias em consonância com a promoção pública de suas imagens, nomes, rostos, sucessos ou, em outro sentido, solicitavam aos visitantes, como a qualquer transeunte informal, a lembrança de seus mortos, o não esquecimento da finitude da vida e, ao mesmo tempo, a fomentação de seus sentimentos de dor e perda. 


\section{Revista de Arqueologia Pública}

\section{AGRADECIMENTOS}

A Pedro Paulo A. Funari, supervisor da pesquisa, a Thiago E. A. Motta que, além da agradável companhia em Roma, concedeu-nos as fotos da Pirâmide de Caius Cestius, bem como a Suiany Bueno Silva.Mencionamos, ainda, o apoio da UFG, Unicamp, CNPq e Capes. No entanto, as reflexões desenvolvidas no decorrer do artigo são de responsabilidade da autora.

\section{REFERÊNCIAS BIBLIOGRÁFICAS}

\section{Documentação Epigráfica:}

SARTORI, A.. Guida alla sezione epigrafica delle raccolte archeologiche di Milano. Milano: Comuni di Milano, 1994.

\section{Documentação Textual:}

HORACE. Odes et Epodes. Les Belles Lettres: Paris, 2002.

LIVIO, Tito. Histoire Romaine. Tome I - Livre I. Texte établi par Jean Bayet e traduit par Gaston Baillet. Paris: Société d’Édition “Les Belles Letres” 1947.

PETRÔNIO. Satyricon. Trad. Cláudio Aquati. São Paulo: Cosac Naify, 2008.

SUETONIUS. The lives of the Caesars (vol. I). Translated by J. C. Rolfe. London: LoebClassical Library, 1979.

\section{Obras Gerais:}

BERSANI, Silvia G. Epigrafia e storia di Roma. Roma: Carocci, 2014.

BIRK, Stine. Man or woman. Cross-gendering and individuality on third century Roman sarcophagi. In: ELSNER, Jás; HUSKINSON, Janet (Ed.). Life, death and representation.Some new work on Roman sarcophagi. New York\Berlin: Walter de GruyterGmbh\& Co. KG, 2011, p. 229-260.

CANDAU, Joël. Memória e Identidade. SãoPaulo:Contexto,2011.

CAPORUSSO, D.; DONATI, M. T.; MASSEROLI, S.; TIBILETTI, T. Immaginidimediolanum. Archeologia e storiadi Milano das V secoloa.C. al V secolod.C. Milano: Comunedi Milano, 2007.

CENERINI, F. La donna romana. Modelli e realtà. Roma: SocietàEditriceilMulino, 2009.

CONSTANTINO, Cláudia. Foro romano, palatino, Coliseo. Milano: Mondadori Electa, 2014.

EDER, Walter. Augustus and the Power of Tradition. In: GALINSKY, Karl (ed.) The Cambridge Companion to the Age of Augustus. Cambridge: Cambridge University Press, 2005, p. 13-32.

ELIAS, Norbert. A solidão dos moribundos. Seguido de envelhecer e morrer. Rio de Janeiro: Zahar, 2001.

FOUCAULT, Michel. A ordem do discurso. São Paulo: Loyola, 1998.

\begin{tabular}{l|l|l|l|l|l|l|} 
(C) Rev. Arqueologia Pública & Campinas, SP & v. 9 & n. 2 & p. 19-29 & DEZ. 2015 & ISSN 2237-8294
\end{tabular}




\section{Revista de Arqueologia Pública}

GALINSKY, Karl (ed.). The Cambridge Companion to the Age of Augustus. Cambridge: Cambridge University Press, 2005.

GENZOR, G. S..Mors: una imagen esquiva. In: SIMÓN, F. M.; POLO, F. P.; RODRÍGUEZ, J. Remesal.. Formae mortis: el tránsito de la vida a la muerte en las sociedades antiguas. Barcelona: Publicacionsi Edicions, 2007, p. 253-282.

GOWING, Alain M. Empire and memory: the representation of the Roman Republic in imperial culture. Cambridge: Cambridge University Press, 2005.

HALES, Shelley. The house and the construction of memory. In: HALES, Shelley. The Roman house and social identity. Cambridge: University Press Cambridge, 2009, p. 40-60.

HIDALDO DE LA VEGA, M. J. Lasemperatrices romanas. Sueños de púrpura y poder oculto. Salamanca. Universidad Salamanca, 2012.

HOPE, Valerie M. Remembering to mourn personal mementos of the dead in Ancient Rome. In: HOPE, Valerie M.; HUSKINSON, Janet (orgs.). Memory and Mourning: Studies on Roman Death. Oxford: Oxbow Books, 2011, pp. 176\195.

Roman Death: the Dying and the Dead in Ancient Rome. New York: Continuum, 2009.

Death in ancient Rome. London and New York: Routledge, 2007.

Status and identity in the Roman world. In: HUSKINSON, Janet. Experiencing Rome.Culture, identity and power in the Roman Empire. London, New York: Routledge, 2005, pp. 125- 152.

Remembering Rome. Memory, funerary monuments and the Roman soldier. In: WILLIAMS, $\mathrm{H}$. Archaeologies of remembrance. Death and memory in past societies. New York: Kluwer Academic, 2003, pp. 113-140.

HUSKINSON, J..Constructing childhood on roman funerary memorials. Hesperia Supplements, vol. 41, Italy, 2007, pp. 323-338.

Picturing the Roman Family. In: RAWSON, Beryl (ed.). A Companion to the Families in the Greekand Roman Worlds. Malden: Blackwell Publishing, 2011. pp. $521-541$

Bad deathes, better memories. In: HOPE, Valerie M.; HUSKINSON, Janet (Ed.). Memory and Mourning: Studies on Roman Death. Oxford: Oxbow Books, 2011, pp.113-149.

LEVARIO, Ana Tania Romero. Transformación y permanencia de las costumbres funerarias del pueblo de San Lorenzo Tezonco. Vita Brevis, Año 1, núm.1, Enero-Junio, pp. 28-40, 2012.

METCALF, Peter; HUNTINGTON, Richard. Celebrations death. The anthropology of mortuary ritual. Cambridge: Cambridge University Press, 2010.

MORIN, Edgar. El ser humano y a muerte. Barcelona: Kairós, 2003.

POLO, Francisco Pina. Eminent corpses: roman aristocracy's passing from life to history. In: SIMÓN, F. M.; POLO, F. P.; RODRÍGUEZ, J. Remesal. Forma e mortis: el tránsito de la vida a la muerte en las sociedades antiguas. Barcelona: PublicacionsiEdicions, 2007, p. 89-100.

ROWE, Gregory. Reconsidering the auctoritas of Augustus. Journal of Roman Studies, v. 103, November, pp. 01-15, 2013.

WALLACE-HADRILL, Andrew. Rome's Cultural Revolution. London: Cambridge, 2008.

\begin{tabular}{l|l|l|l|l|l|l|} 
(C) Rev. Arqueologia Pública & Campinas, SP & v. 9 & n. 2 & p. 19-29 & DEZ. 2015 & ISSN 2237-8294
\end{tabular}


WOOLF, Greg. Literacy or literacies in Rome? In: JOHNSON, William A.; PARKER, Holt N. (Ed.). Ancient Literacies.The Culture of Reading in Greece and Rome. Oxford: Oxford University Press, 2009, pp. 46-68. 